\title{
A Proof of Brane Creation via $M$-theory
}

\author{
Toshio Nakatsu, Kazutoshi Ohta, Takashi Yokono and Yuhsuke Yoshida \\ Department of Physics, \\ Graduate School of Science, Osaka University, \\ Toyonaka, Osaka 560, JAPAN
}

\begin{abstract}
We study configurations of a single $M$ fivebrane in the geometry $\mathbf{R}^{7} \times Q$ where $Q$ is the Taub-NUT space. Taking the IIA limit at each value of their modulus, two possibilities of the IIA configuration are revealed. One consists of a NS fivebrane and a D sixbrane while the other consists of a NS fivebrane, a D sixbrane and a D fourbrane. In the latter case the fourbrane is shown to be suspended by the fivebrane and the sixbrane. This appearance of fourbrane can be interpreted in Type IIA picture as the result of the crossing of the fivebrane and the sixbrane.
\end{abstract}


The possibility of brane creation in string theory was first pointed out in [1]. Several proofs for it have been given [2], [3], [4], [5] mainly based on the so-called anomaly-inflow argument [6] and $\mathrm{M}$ (atrix) theory description [7]. In this paper we would like to add another proof of brane creation based on a different perspective. Namely we examine configurations of a M fivebrane in the eleven-dimensional geometry $\mathbf{R}^{7} \times Q$ where $Q$ is the Taub-NUT space. This elevendimensional background is the classical solution of eleven-dimensional SUGRA which provides [8] a single D sixbrane solution of IIA string theory. In these configurations two-dimensional part of the worldvolume of fivebrane is embedded holomorphically into $Q$. With a detailed study on its holomorphic embedding we consider its IIA limit at each value of the modulus of the configurations. It is shown that there are two possibilities of the IIA configuration, one of which is the configuration consisting of a NS fivebrane and a D sixbrane while the other is the configuration consisting of a NS fivebrane, a D sixbrane and a D fourbrane. In the latter case the fourbrane is actually suspended between the fivebrane and the sixbrane. This appearance of fourbrane can be interpreted as the result of the crossing of the fivebrane and the sixbrane in IIA picture.

Consider a single D sixbrane with worldvolume $\left(x^{0}, x^{1}, x^{2}, x^{3}, x^{7}, x^{8}, x^{9}\right)$. D sixbrane is a magnetic source of the $U(1)$ gauge potential $A$ which is a bosonic ingredient of IIA SUGRA multiplet. Notice that, compactifying the eleventh dimension into a circle, the eleven-dimensional metric tensor $G_{M N}(0 \leq M, N \leq 10)$, which is a bosonic ingredient of eleven-dimensional SUGRA multiplet, gives rise to the $U(1)$ gauge potential $A$. Therefore $\mathrm{D}$ sixbrane is the Kaluza-Klein monopole. Though the four-dimensional space $\left(x^{4}, x^{5}, x^{6}, x^{10}\right)$ transversal to the sixbrane is originally a flat space $\mathbf{R}^{3} \times S^{1}\left(\equiv Q_{0}\right)$, the existence of sixbrane makes it a curved one. It actually becomes [8] the Taub-NUT space $Q$. To describe the Taub-NUT geometry let us introduce the coordinates $(\vec{r}, \sigma)$

$$
\vec{r} \equiv\left(2 x^{4} / R, 2 x^{5} / R, 2 x^{6} / R\right) \quad, \quad \sigma \equiv 2 x^{10} / R
$$

where $R$ is the radius of $S^{1}$. The Taub-NUT metric acquires the standard form [9]

$$
d s^{2}=\frac{V}{4} d \vec{r}^{2}+\frac{1}{4 V}(d \sigma+\vec{\omega} \cdot d \vec{r})^{2},
$$


where

$$
V=1+\frac{1}{\left|\vec{r}-\vec{r}_{0}\right|}
$$

$\vec{r}_{0}$ denotes the position of the sixbrane. And $\vec{\omega}$ is chosen so that it satisfies the relation

$$
\vec{\nabla} \times \vec{\omega}=\vec{\nabla} V
$$

Notice that the $U(1)$ gauge potential $A$ can be read from eq.(2) as $A=\vec{\omega} \cdot d \vec{r}$. Since $\vec{\omega}$ is a quantity determined by relation (丑), it has an ambiguity depending on the direction of the Dirac string associated with the sixbrane. It turns out useful to fix the gauge potential including the Dirac string. By separating the three-vector $\vec{r}$ into two parts $\vec{r}=(v, b)$ where

$$
v \equiv \frac{2\left(x^{4}+i x^{5}\right)}{R}, b \equiv \frac{2 x^{6}}{R}
$$

the $U(1)$ gauge potential $A$ can be put into the form

$$
A=\operatorname{Im}\{\delta d v\}
$$

where we introduce $\delta$ as

$$
\delta \equiv \frac{b+b_{0}+\sqrt{\left(b+b_{0}\right)^{2}+\left|v+e_{0}\right|^{2}}}{\sqrt{\left(b+b_{0}\right)^{2}+\left|v+e_{0}\right|^{2}}} \frac{1}{\left|v+e_{0}\right|}
$$

Notice that the position of the sixbrane is denoted by $\vec{r}_{0}=\left(-e_{0},-b_{0}\right)$ in eq. (7). With the above choice of the gauge potential the field strength $F=d A$ acquires the Dirac string of the following type

$$
F \sim \pi i \theta\left(b+b_{0}\right) \delta^{(2)}\left(v+e_{0}\right) d v \wedge d \bar{v}
$$

The Dirac string is now realized as the semi-infinite line in the $(v, b)$-space which runs from $\left(-b_{0},-e_{0}\right)$, parallel with the $b$-axis, into $\left(+\infty,-e_{0}\right)$. What is a counterpart of the Dirac string in the Taub-NUT geometry? Recall that the Taub-NUT space can be regarded as the fibered space which base and fiber are respectively the $(v, b)$-space and $S^{1}$. There exists a singular fiber at $(v, b)=\left(-e_{0},-b_{0}\right)$. This is the so-called NUT singularity. With this understanding of the Taub-NUT space, an inverse image of the Dirac string in the Taub-NUT space gives its counterpart. It is the non-compact two-cycle which is precisely projected onto the Dirac string. 
The Taub-NUT space $Q$ admits to have an hyper-Kähler structure. Especially it is possible to choose a complex structure so that the Taub-NUT metric (2) becomes Kähler. It is given [10] by introducing the following holomorphic coordinates $(v, y)$

$$
\begin{aligned}
& v=\frac{2\left(x^{4}+i x^{5}\right)}{R}, \\
& y \equiv e^{-\frac{b+i \sigma}{2}}\left(-b-b_{0}+\sqrt{\left(b+b_{0}\right)^{2}+\left|v+e_{0}\right|^{2}}\right)^{\frac{1}{2}} .
\end{aligned}
$$

With these holomorphic coordinates the Taub-NUT metric (2) t can be rewritten as

$$
d s^{2}=\frac{V}{4} d v d \bar{v}+\frac{1}{4 V}\left(\frac{2 d y}{y}-\delta d v\right) \overline{\left(\frac{2 d y}{y}-\delta d v\right)} .
$$

So it becomes [10 Kählerian.

Let us consider a $\mathrm{M}$ fivebrane which worldvolume is topologically $\mathbf{R}^{4} \times \mathbf{C}$. Its fourdimensional part " $\mathbf{R}$ " is mapped into the $\left(x^{0}, x^{1}, x^{2}, x^{3}\right)$-space and identified with it. Its two-dimensional part denoted by the complex plane is supposed to be holomorphically embedded into the above Taub-NUT space. Let us consider the holomorphic embedding of the following type

$$
y=v+e_{0},
$$

where " $y$ " is the complex function of $v, b$ and $\sigma$ given in eq.(10). As we shall show soon, eq.(12) describes a configuration consisting of the fivebrane sitting to the right of the sixbrane in the Taub-NUT space. Using expression (10) one can separate eq.(12) into radial and angular parts. The radial part acquires the form

$$
e^{-b}=b+b_{0}+\sqrt{\left(b+b_{0}\right)^{2}+\left|v+e_{0}\right|^{2}},
$$

while the angular part becomes

$$
e^{-i \frac{\sigma}{2}}=\frac{v+e_{0}}{\left|v+e_{0}\right|} .
$$

Notice that these two equations determine respectively $b$ and $\sigma$ as the functions of $v$ and $\bar{v}$. Let us denote them by $b(v)$ and $\sigma(v)$ for brevity.

\footnotetext{
${ }^{1} A=\vec{\omega} \cdot d \vec{r}$ has the form given in eq. (6).
} 
We shall first examine the solution $\sigma(v)$ for eq.(14). It actually describes a vortex at $v=-e_{0}$. This vortex corresponds to an intersection of the Dirac string of the $U(1)$ gauge potential $A$ and the fivebrane 2 . It can be explained by the following argument : Consider the sigma model action of the fivebrane. Its bosonic part will include the term, $\int d^{6} \xi \sqrt{|h|} h^{i j} \partial_{i} X^{M} \partial_{j} X^{N} G_{M N}(X)$, where $\xi^{i}(0 \leq i \leq 5)$ is the worldvolume coordinates and $h_{i j}$ is an auxiliary metric on the worldvolume. By rewriting [14] the eleven-dimensional metric $G_{M N}$ under the $S^{1}$-compactification in terms of the ten-dimensional quantities $\left(g_{m n}, A_{m}, e^{\phi}\right)(0 \leq m, n \leq 9)$, it can be seen that the above bosonic part induces the term, $\int d^{6} \xi \sqrt{|h|} e^{\frac{4}{3} \phi} h^{i j} \nabla_{i} X^{10} \nabla_{j} X^{10}$, where $\nabla_{i} X^{10} \equiv$ $\partial_{i} X^{10}+\partial_{i} X^{m} A_{m}(X)$. This gives an interaction of $X^{10}(\sim \sigma)$ with the $U(1)$ gauge potential. Through this interaction the Dirac string will make a vortex (of $\sigma$ ) on the fivebrane worldvolume.

It should be also noticed that, since the sixbranes are the magnetic sources of the $U(1)$ gauge potential, the number of the vortices made by the intersection between the Dirac strings (or their non-compact two-cycles) and the fivebranes define [11] the linking number [1] among them. As for the configurations determined by $(\sqrt{12})$ it is an invariant.

Next let us pay attention to the solution $b(v)$ for eq.(13). The above argument makes the fivebrane intersect with the Dirac string which we have just fixed in eq.(8). Therefore it implies the inequality

$$
\left.b(v)\right|_{v=-e_{0}}>-b_{0}
$$

which means that the fivebrane is located on the "right" of the sixbrane in the Taub-NUT space.

At this stage it might be convenient to describe a process of brane creation in a very heuristic manner ?. For this purpose we shall consider the holomorphic embedding of the fivebrane when the position of the sixbrane $\vec{r}_{0}=\left(-e_{0},-b_{0}\right)$ goes to infinity. Let us examine the situations, $-b_{0} \rightarrow \pm \infty$. We first remark that, in the region $\left|v+e_{0}\right| \ll\left|b+b_{0}\right|$, the R.H.S of eq. (13)

\footnotetext{
${ }^{2}$ It is the intersection in the $(v, b)$-space. To discuss their intersection in the Taub-NUT space we should replace the Dirac string by the non-compact two-cycle projected to the Dirac string. But, even in the Taub-NUT space, formulas described in terms of differential forms such as eq.(8) do not change.

${ }^{3} \mathrm{~A}$ similar observation, but in a flat background, was also made in [12]. We thank the authors of [12] for letting us know about it.
} 
behaves approximately as

$$
b+b_{0}+\sqrt{\left(b+b_{0}\right)^{2}+\left|v+e_{0}\right|^{2}} \sim\left(b+b_{0}+\left|b+b_{0}\right|\right)+\frac{\left|v+e_{0}\right|^{2}}{2\left|b+b_{0}\right|} .
$$

Owing to this estimate, taking care of the absolute value in it, eq.(13) will acquire the following form at the limit $-b_{0} \rightarrow+\infty$

$$
e^{-\frac{b}{2}}=\text { const. }\left|v+e_{0}\right|
$$

while it will become at the limit $-b_{0} \rightarrow-\infty$

$$
e^{-\frac{b}{2}}=\text { const. }
$$

Notice that the Taub-NUT space itself can be regarded as the flat space $Q_{0}=\mathbf{R}^{3} \times S^{1}$ in these limits. So, according to the discussion given in [13], the first case describes, in IIA picture, a configuration consisting of a NS fivebrane and a semi-infinite D fourbrane sticking to the NS fivebrane from the right while the second case describes a configuration of a NS fivebrane. This will mean that the sixbrane running from $x^{6}=-\infty$ to $x^{6}=+\infty$ creates a $\mathrm{D}$ fourbrane by crossing the fivebrane. This may give an heuristic explanation for the brane creation via M theory. In spite of its intuitiveness it is still too naive to justify. So let us provide a rigorous treatment.

\section{Detail on M fivebrane}

Without loss of generality we can set the position of the sixbrane $\vec{r}_{0}=0$ by a parallel shift of the coordinates. In such coordinates eq.(13) acquires the form

$$
e^{-b}=A\left(b+\sqrt{b^{2}+|v|^{2}}\right)
$$

where $A \equiv e^{-b_{0}}$. Since it does not depend on the phase of $v$ the solution for this equation is a function of $|v|$ and $b_{0}$. Let us denote it by $b\left(|v| ; b_{0}\right)$. Notice that relation (15) implies

$$
\beta\left(b_{0}\right) \equiv b\left(0 ; b_{0}\right)>0 \text {. }
$$

Let us start by describing how the solution depends on $|v|$ and $b_{0}$. It can be given by partiallydifferentiating eq.(19) with respect to $|v|$ and $b_{0}$. After a little calculation we obtain

$$
\begin{aligned}
\frac{\partial b\left(|v| ; b_{0}\right)}{\partial|v|} & =-\frac{1}{1+\Delta\left(|v| ; b_{0}\right)} \frac{-b\left(|v| ; b_{0}\right)+\Delta\left(|v| ; b_{0}\right)}{|v|} \\
\frac{\partial b\left(|v| ; b_{0}\right)}{\partial b_{0}} & =\frac{\Delta\left(|v| ; b_{0}\right)}{1+\Delta\left(|v| ; b_{0}\right)}
\end{aligned}
$$


where we introduce $\Delta\left(|v| ; b_{0}\right)$ by

$$
\Delta\left(|v| ; b_{0}\right) \equiv \sqrt{b\left(|v| ; b_{0}\right)^{2}+|v|^{2}}
$$

Since it holds $\partial b\left(|v| ; b_{0}\right) / \partial|v|<0$ for $|v|>0$ and $\partial b\left(|v| ; b_{0}\right) / \partial b_{0}>0$, it means that $b\left(|v| ; b_{0}\right)$ is monotonically decreasing with respect to $|v|$ while it is monotonically increasing with respect to $b_{0}$.

Let us consider the behavior of $\beta\left(b_{0}\right)=b\left(0 ; b_{0}\right)$. Eq.(22) implies the following 1 st order differential equation for $\beta$

$$
\frac{d \beta\left(b_{0}\right)}{d b_{0}}=\frac{\beta\left(b_{0}\right)}{1+\beta\left(b_{0}\right)},
$$

which can be integrated out. One can find $e^{b_{0}}=C \beta\left(b_{0}\right) e^{\beta\left(b_{0}\right)}$ where $C$ is a positive constant. Owing to this form there exists a critical value $b_{0}^{*}$ such that $b_{0} \leq b_{0}^{*}$ implies $\beta\left(b_{0}\right) \geq b_{0}$ and vice versa. In such a case, since it has been pointed out that $b\left(|v| ; b_{0}\right)$ is monotonically decreasing with respect to $|v|$, there exists $r\left(b_{0}\right)$ which satisfies $b\left(r\left(b_{0}\right) ; b_{0}\right)=b_{0}$. An explicit form of $r\left(b_{0}\right)$ can be obtainable from eq.(19) by inserting $(|v|, b)=\left(r\left(b_{0}\right), b_{0}\right)$ into it

$$
r\left(b_{0}\right)=\sqrt{1-2 b_{0}}
$$

Since $r\left(b_{0}\right)$ is, by definition, a non-negative quantity and it must be zero at the critical value of $b_{0}$, we can find out $b_{0}^{*}$ as

$$
b_{0}^{*}=1 / 2 .
$$

Notice that it holds $\beta\left(b_{0}^{*}\right)=b_{0}^{*}$. Inserting this value into the integrated form $e^{b_{0}}=C \beta\left(b_{0}\right) e^{\beta\left(b_{0}\right)}$, we obtain $C=2$. Hence $\beta\left(b_{0}\right)$ is determined by the relation

$$
2 \beta\left(b_{0}\right) e^{\beta\left(b_{0}\right)}=e^{b_{0}}
$$

Finally we shall consider the intersection between the fivebrane and $b=0$ plane in the Taub-NUT space. It is a circle with a definite radius. The radius $u\left(b_{0}\right)$ can be read from eq. (19). It is given by

$$
u\left(b_{0}\right)=e^{b_{0}} .
$$




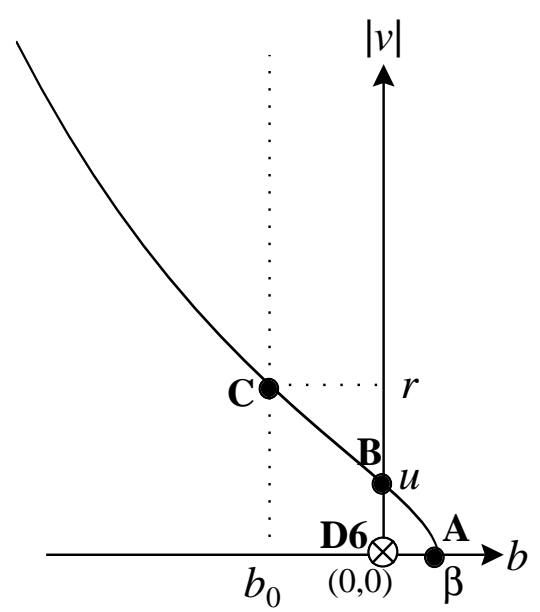

(a) $b_{0} \leq 0$

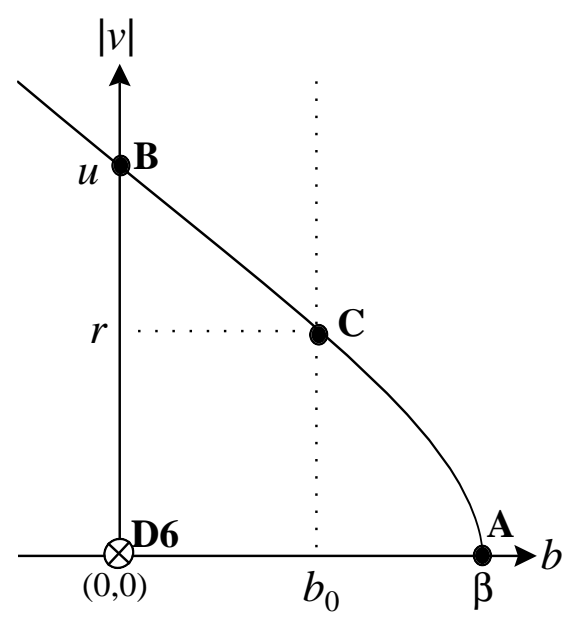

(b) $0<b_{0} \leq 1 / 2$

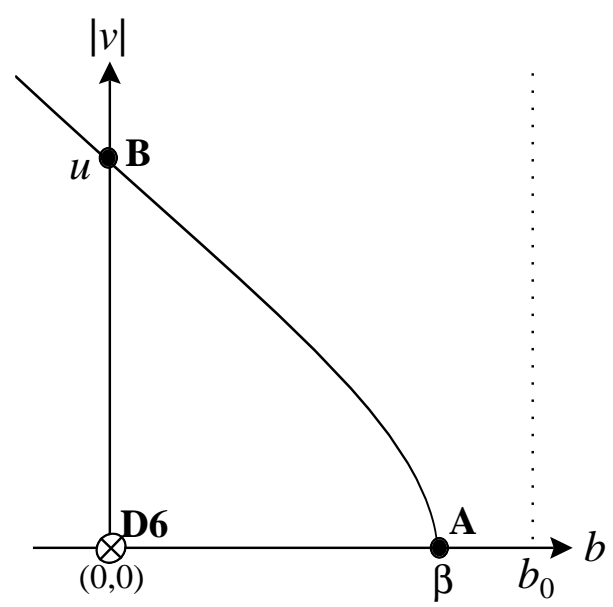

(c) $1 / 2<b_{0}$

Figure 1: Shapes of the fivebrane in the $(|v|, b)$-plane

Now, gathering all these data about some characteristic points of the embedded two-plane, it is possible to draw the shape of the fivebrane in the Taub-NUT space. There appear three cases depending on the values of $b_{0}: b_{0} \leq 0,0<b_{0} \leq 1 / 2$ and $b_{0}>1 / 2$. In Fig.1 these three cases are depicted in the $(|v|, b)$-plane. Three characteristic points are denoted by A, B and C. Their coordinates in the $(|v|, b)$-plane are respectively $\left(0, \beta\left(b_{0}\right)\right),\left(u\left(b_{0}\right), 0\right)$ and $\left(r\left(b_{0}\right), b_{0}\right)$.

\section{IIA limit}

Generically the IIA limit of the above configurations will be achieved by taking the limit $R \rightarrow 0$ with fixing the value of $R b_{0} / 2$ f 15$]$. (Therefore $b_{0}$ goes to infinity.) Let us denote this value by $x_{0}^{6}$. We first examine the case of $b_{0} \leq 0$. In this case one should set $x_{0}^{6} \leq 0$ because of the relation $x_{0}^{6}=R b_{0} / 2$. And, to fix $x_{0}^{6}$ at a finite negative value, one must take $b_{0} \rightarrow-\infty$. Let us consider the IIA limits of the points A, B and C. The point A will become the point given by the coordinates $\left(\left|x^{4}+i x^{5}\right|, x^{6}\right)=\left(0, \lim _{R \rightarrow 0} R \beta\left(b_{0}\right) / 2\right)$. What is the value of $\lim _{R \rightarrow 0} R \beta\left(b_{0}\right)$ ? We shall first rewrite eq.(27) into the form

$$
R \beta\left(b_{0}\right)+R \ln R \beta\left(b_{0}\right)-R \ln 2 R=2 x_{0}^{6} .
$$

\footnotetext{
${ }^{4}$ The factor $1 / 2$ is our convention.
} 
Notice that, owing to eq. (20), $R \beta\left(b_{0}\right)$ is a positive quantity at a non-zero value of $R$. Therefore, in order to fix the R.H.S. of the equation at a negative value, $R \ln R \beta\left(b_{0}\right)$ in the L.H.S. must become a negative quantity as $R$ goes to zero. This implies $\lim _{R \rightarrow 0} R \beta\left(b_{0}\right)=0$. So the point A approaches to the origin. Next we examine the point B. It becomes the point given by the coordinates $\left(\left|x^{4}+i x^{5}\right|, x^{6}\right)=\left(\lim _{R \rightarrow 0} R u\left(b_{0}\right) / 2,0\right)$. Using the expression given in (28) one can also see $\lim _{R \rightarrow 0} R u\left(b_{0}\right)=0$. Hence, the point $\mathrm{B}$ also approaches to the origin under the IIA limit. As regards the point $\mathrm{C}$, It becomes the point given by the coordinates $\left(\left|x^{4}+i x^{5}\right|, x^{6}\right)=$ $\left(\lim _{R \rightarrow 0} \operatorname{Rr}\left(b_{0}\right) / 2, x_{0}^{6}\right)$. By the expression given in (25) one can see $\lim _{R \rightarrow 0} \operatorname{Rr}\left(b_{0}\right)=0$. So, the point $\mathrm{C}$ approaches to $\left(0, x_{0}^{6}\right)$.

The segment between the points $\mathrm{A}$ and $\mathrm{C}$ on the fivebrane in the $(|v|, b)$-plane reduces, in the IIA limit, to a line segment $\left(0, x^{6}\right)$ where $x_{0}^{6} \leq x^{6} \leq 0$. Notice that this line segment in the $\left(\left|x^{4}+i x^{5}\right|, x^{6}\right)$-plane is still one-dimensional in the three-dimensional $\left(x^{4}, x^{5}, x^{6}\right)$-space since $\left|x^{4}+i x^{5}\right|=0$ implies $x^{4}=x^{5}=0$. How about the other part of the fivebrane? To obtain its IIA limit let us rewrite eq.(21) in terms of $x^{6}$ and $\left|x^{4}+i x^{5}\right|$. By introducing the quantity $t=b /|v|\left(=x^{6} /\left|x^{4}+i x^{5}\right|\right)$ it becomes

$$
\begin{aligned}
\frac{\partial x^{6}}{\partial\left|x^{4}+i x^{5}\right|} & =\lim _{R \rightarrow 0} R \frac{t-\sqrt{1+t^{2}}}{R+\left|x^{4}+i x^{5}\right| \sqrt{1+t^{2}}} \\
& =0 .
\end{aligned}
$$

So, this part of the fivebrane approaches to the line $\left(\left|x^{4}+i x^{5}\right|, x_{0}^{6}\right)$. Due to the rotational symmetry around the $x^{6}$-axis this line corresponds to a two-dimensional plane in the $\left(x^{4}, x^{5}, x^{6}\right)$ space. Therefore the IIA limit of the configuration with $b_{0} \leq 0$ describes the configuration of a NS fivebrane, a D fourbrane and a D sixbrane. The NS fivebrane is located at $\left(x^{6}, x^{7}, x^{8}, x^{9}\right)=$ $\left(x_{0}^{6}, 0,0,0\right)$ while the $\mathrm{D}$ fourbrane with worldvolume $\left(x^{0}, x^{1}, x^{2}, x^{3}, x^{6}\right)$ is suspended, in the $\left(x^{4}, x^{5}, x^{6}\right)$-space, between the point $\left(x^{4}, x^{5}, x^{6}\right)=\left(0,0, x_{0}^{6}\right)$ on the NS fivebrane and the D sixbrane at $\left(x^{4}, x^{5}, x^{6}\right)=(0,0,0)$. (Fig.2 (a))

Next we shall consider the case of $0<b_{0} \leq 1 / 2$. In this case we must set $x_{0}^{6}>0$. So, $b_{0} \rightarrow+\infty$. The IIA limit of the point $\mathrm{A}$ is given by the coordinates $\left(\left|x^{4}+i x^{5}\right|, x^{6}\right)=$ $\left(0, \lim _{R \rightarrow 0} R \beta\left(b_{0}\right) / 2\right)$. Let us show $\lim _{R \rightarrow 0} R \beta\left(b_{0}\right)=x_{0}^{6}$. Rewrite eq. (27) into the form

$$
R\left(\beta\left(b_{0}\right)+\ln 2 \beta\left(b_{0}\right)\right)=2 x_{0}^{6}
$$

In order to fix the R.H.S. of the equation at a positive value, $\beta+\ln 2 \beta$ in the L.H.S. must 


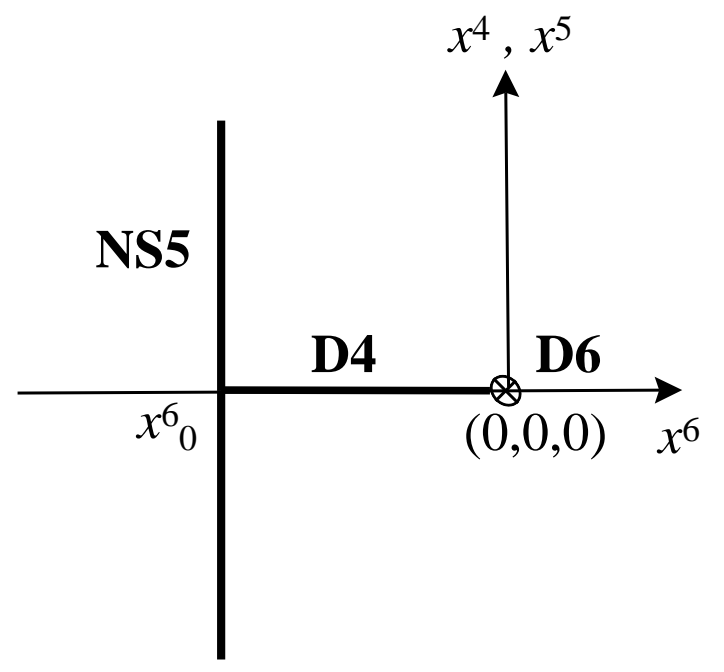

(a)

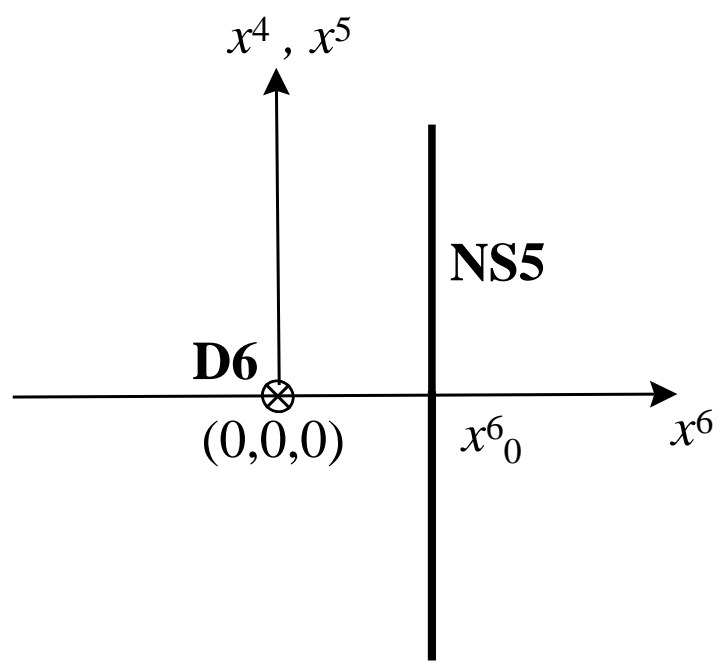

(b)

Figure 2: IIA limits of M-theory brane configurations depicted in Fig.1 : (a) D fourbrane is suspended between NS fivebrane and D sixbrane. (b) There are only NS fivebrane and D sixbrane.

approach to $+\infty$ as $R$ goes to zero. In particular it holds $\beta \rightarrow+\infty$. Hence $\lim _{R \rightarrow 0} \ln \beta / \beta=0$. Regarding the L.H.S. of eq.(31) as $R \beta\left(b_{0}\right)\left(1+\ln 2 \beta\left(b_{0}\right) / \beta\left(b_{0}\right)\right)$ and considering its IIA limit, we obtain $\lim _{R \rightarrow 0} R \beta\left(b_{0}\right) / 2=x_{0}^{6}$. Therefore the point A approaches to $\left(0, x_{0}^{6}\right)$ in the IIA limit. As for the point $\mathrm{C}$, it becomes the point given by the coordinates $\left(\lim _{R \rightarrow 0} \operatorname{Rr}\left(b_{0}\right) / 2, x_{0}^{6}\right)$. With the same reason as in the previous case it is $\left(0, x_{0}^{6}\right)$ in the IIA limit. Finally the point B becomes the point given by the coordinates $\left(\lim _{R \rightarrow 0} R u\left(b_{0}\right) / 2,0\right)$. Since $R u\left(b_{0}\right)=2 R \beta\left(b_{0}\right) e^{R \beta\left(b_{0}\right) / R}$ behaves as $2 x_{0}^{6} e^{x_{0}^{6} / R}$ at a small value of $R$ and goes to the infinity after the IIA limit, it holds $\lim _{R \rightarrow 0} R u\left(b_{0}\right)=+\infty$. To summarize, the IIA limit of the configuration with $0<b_{0} \leq 1 / 2$ describes the configuration of a NS fivebrane and a D sixbrane. The NS fivebrane is located at $\left(x^{6}, x^{7}, x^{8}, x^{9}\right)=\left(x_{0}^{6}, 0,0,0\right)$ and the $\mathrm{D}$ sixbrane is at $\left(x^{4}, x^{5}, x^{6}\right)=(0,0,0)$. There appears no D fourbrane. (Fig.2 (b))

Finally the study for the case of $b_{0}>1 / 2$ is remained. But it is easy to see that this case reduces to that of $0<b_{0} \leq 1 / 2$. In particular the IIA limit coincides with the previous case. 


\section{Acknowledgments}

T.N. is supported in part by Grant-in-Aid for Scientific Research 08304001. K.O. and Y.Y. are supported in part by the JSPS Research Fellowships.

\section{References}

[1] A. Hanany and E. Witten, "Type IIB Superstrings, BPS Monopoles and ThreeDimensional Gauge Dynamics", Nucl. Phys. B492 (1997) 152, hep-th/9611230.

[2] C.P. Bachas, M.R. Douglas and M.B. Green, "Anomalous Creation of Branes", hepth/9705074.

S.P. de Alwis, "A Note on Brane Creation", hep-th/9706142.

[3] U. Danielsson, G. Ferretti and I.R. Klebanov, "Creation of Fundamental Strings by Crossing D-branes", Phys. Rev. Lett. 79 (1997) 1984, hep-th/9705084.

O. Bergman, M.R. Gaberdiel and G. Lifschytz, "Branes, Orientifolds and the Creation of Elementary Strings", hep-th/9705130.

[4] P. Ho and Y. Wu, "Brane Creation in M(atrix) theory", hep-th/9708137.

N. Ohta, T. Shimizu and J-G. Zhou, "Creation of Fundamental String in M(atrix) theory", hep-th/9710218.

[5] Y. Imamura, "D-particle creation on an orientifold plane", hep-th/9710026.

[6] M. Green, J.A. Harvey and G. Moore, "I-Brane Inflow and Anomalous Couplings on DBranes", Class. Quant. Grav. 14 (1997) 47, hep-th/9605033.

J.M. Izquierdo and P.K. Townsend, "Axionic defect anomalies and their cancellation", Nucl. Phys. B414 (1994) 93.

J. Blum and J.A. Harvey, "Anomaly inflow for gauge defects", Nucl. Phys. B416 (1994) 119.

[7] T. Banks, W. Fischler, S.H. Shenker and L. Suskind, "M theory as A Matrix Model : A Conjecture", hep-th/9610043. 
[8] P.K. Townsend, "The eleven-dimensional supermembrane revisited", Phys. Lett. B350 (1995) 184, hep-th/9501068.

[9] S.W. Hawking, "Gravitational Instantons", Phys. Lett. 60A (1977) 81.

G.W. Gibbons and S.W. Hawking, "Classification of Gravitational Instanton Symmetries", Commun. Math. Phys. 66 (1979) 291.

[10] T. Nakatsu, K. Ohta, T. Yokono and Y. Yoshida, "Higgs Branch of $N=2$ SQCD and $M$ theory Branes", hep-th/9707258.

[11] R. Bott and L.W. Tu, "Differential Forms in Algebraic Topology", Springer-Verlag New York, 1982.

[12] M. Schmaltz and R. Sundrum, "N=1 Field Theory Duality from M Theory", hepth/9708015.

[13] E. Witten, "Solutions of Four-Dimensional Field Theories via M Theory", hep-th/9703166.

[14] M.J. Duff, P.S. Howe, T. Inami and K.S. Stelle, "Superstrings in $D=10$ From Supermembrane In $D=11 "$ ", Phys. Lett. B191 (1987) 70.

[15] A. Brandhuber, N. Itzhaki, V. Kaplunovsky, J. Sonnenschein and S. Yankielowicz, "Comments on the M theory Approach to $N=1$ SQCD and Brane Dynamics", hep-th/9706127. 